\title{
Refresher: Ohrakupunktur
}

\section{Geschichte}

Bereits in der Antike wusste man um die Therapiemöglichkeiten über die Ohrmuschel. So soll Hippokrates mittels einer Blutung am äußeren Ohr versucht haben, Impotenz zu kurieren, ägyptische Seeleute sollen versucht haben, durch Durchstechung des Ohrläppchens ihr Sehvermögen für die Seefahrt zu steigern. Kauterisationen der Ohrmuschel zur Therapie des Ischiasschmerzes sind z. B. durch persische Heiler bekannt.

In Europa finden sich im 17. und 18. Jahrhundert erste Hinweise auf Ischialgiebehandlung mittels Ohrkauterisation, z.B. beschreibt Zaçtus Lusitanus 1637 Kauterisationen am Ohr zur Therapie der Ischialgie in Portugal.

Erst um 1950 jedoch entdeckte der französische Neurologe Paul Nogier das Somatotop auf der Ohrmuschel und erstellte eine umfassende Darstellung der Therapie über die Ohrmuschel. Er fand bei zahlreichen Patienten, die durch eine Heilerin (Mme. Barrin) aufgrund einer Ischialgie therapiert worden waren, Kauterisationsnarben an der Anthelix. Diese Patienten gaben erstaunliche Erfolge dieser Therapie an, sodass Nogier das Phänomen weiter untersuchte. Seine Versuche, bei Rezidiven dieser Beschwerden die drucksensiblen Punkte im Bereich der Anthelix mit Akupunktur (zunächst mit Näh- oder Stecknadeln) zu behandeln, waren erfolgreich. Er gelangte zu der Auffassung, dass sich Störungen des Körpers regelhaft durch empfindliche oder schmerzhafte Punkte an der Ohrmuschel nachweisen ließen. Nogier stellte seine Arbeiten unter dem Namen „Aurikulotherapie“ 1956 auf einem Akupunktur-Kongress in Marseille vor und begründete damit die heutige Ohrakupunktur.

Diese Ergebnisse gelangten 1959 nach China. Hier wurde die Ohrmuschel zwar als wichtige topografische Region, an der sich einige Meridiane der Körperakupunktur treffen, wahrgenommen, eine eigenständige Ohrakupunktur existierte allerdings nicht. Es entwickelte sich eine „chi-

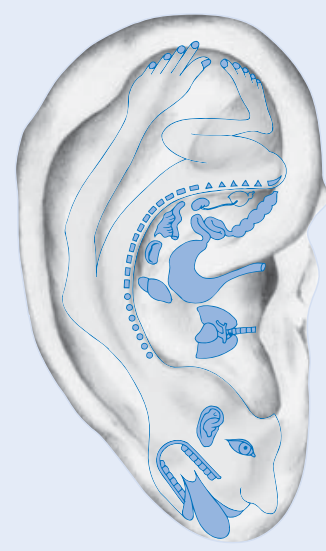

Abb. 1 Embryo-Modell. (c) [2]

nesische Ohrakupunktur“ mit eigener Nomenklatur und Kartografie der Ohrpunkte, die in Europa durch die österreichischen Ärzte Georg König und Ingrid Wancura mit einem Ziffernsystem belegt und interpretiert wurde. Die chinesische Ohrakupunktur hat viele Gemeinsamkeiten mit der Aurikulotherapie von Nogier, teilweise werden jedoch unterschiedliche Punktlokalisationen angegeben. Die Bestrebungen gehen dahin, gesicherte Erkenntnisse beider Richtungen unter dem Dach der angewandten Ohrakupunktur zu integrieren zum Wohle der Patienten.

\section{Repräsentationszonen}

Die Ohrakupunktur stellt eine Sonderform der Akupunktur dar, der das Konzept der Somatotopie zugrunde liegt. Dieser Begriff ist zusammengesetzt aus gr. Soma $=$ Körper und Topos = Ort und meint die Abbildung des Körpers auf ein Areal.

Nogier erstellte eine Kartografie der Ohrmuschel aus der Zusammenschau der Embryogenese und Innervation der Ohrmuschel sowie der Keimblattzuordnung und aus den von ihm erforschten Reflexbeziehungen. Die Repräsentation des Körpers auf der Ohrmuschel erinnert an einen auf dem Kopf stehenden Embryo ( Abb. 1).

Diese Vorstellung erleichtert die Orientierung auf der Ohrmuschel. Unter Zuhilfenahme der beiden durch die anatomi-

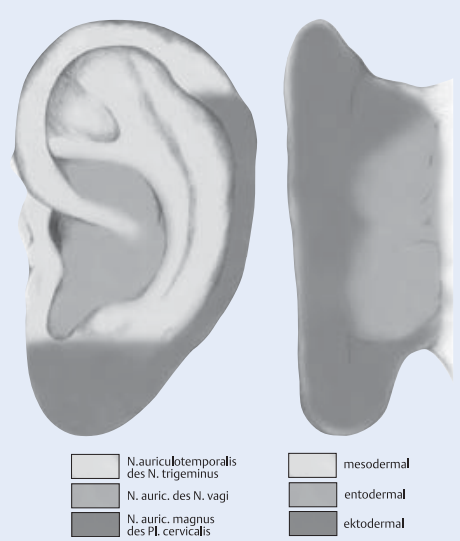

Abb. 2 Die 3 Innervationszonen und ihre embryogenetische Herkunft. (C) [2]

schen Strukturen annähernd vorgegebenen Achsen, der Anthelix (Vertikalachse) als Projektion der Wirbelsäule und Helixwurzel (Horizontalachse) als ZwerchfellLinie, ist die Zuordnung von Repräsentationszonen möglich. Der Kopf mit Sinnesorganen ist kaudal lokalisiert, die Extremitäten dagegen kranial.

Das Ohrmuschelsomatotop ist ein komplexes reflektorisches System. Störungen des Körpers stellen sich als empfindliche Punkte auf der Ohrmuschel dar. Umgekehrt kann über diese Punkte auch regulierend auf die korrespondierenden Körperareale eingewirkt werden.

\section{Embryologie und Innervation}

Auf der Ohrmuschelvorderseite finden sich 3 Innervationsgebiete (C Abb. 2). Beteiligt an der nervalen Versorgung der Ohrmuschel sind in erster Linie

- Nervus auricularis (Ramus d. N. vagus),

- Nervus auricularis magnus (R.d. Plexus cervicalis) und

- Nervus auriculotemporalis (R. d. N. trigeminus).

Auf der Rückseite gesellt sich zusätzlich zu dem R. auricularis des N. vagus und des N. auricularis magnus mit nahezu $50 \%$ der N. occipitalis minor. 
Die Innervationszonen der Ohrmuschel stehen im Zusammenhang mit der embryogenetischen Herkunft der auf ihnen repräsentierten Organe ( $\mathrm{Abb} .2$ ).

- Repräsentationen der entodermal entstandenen Eingeweideorgane liegen in der vom N. vagus innervierten Ebene der Concha.

- Repräsentationen der aus dem Mesoderm entstandenen Bestandteile des Körpers, z.B. Skelett, Muskulatur, Bindegewebe, aber auch Harnleiter und Uterus, liegen in dem vom N. trigeminus innervierten Anteil der Ohrmuschel.

- Die aus dem Ektoderm stammenden Anteile - Gehirn, Nervensystem, Haut und Epithelien der Sinnesorgane - sind dem vornehmlich vom Plexus cervicalis superficialis versorgten Gebiet von Lobulus und Teil des Helixrandes bis in Höhe des Darwin-Höckers zuzuordnen.

\section{Besonderheiten}

Die Ohrakupunkturpunkte sind gut zugänglich, sie liegen ca. $1 \mathrm{~mm}$ tief. Oft tritt die Wirkung, v. a. bei Beschwerden des Bewegungsapparats, rasch ein. Im Gegensatz zu den Körperakupunkturpunkten handelt es sich um Reaktionspunkte, die nur bei einer Funktionsstörung ihres korrespondierenden Organs aktiv und auffindbar sind.

Nicht alle Punkte korrespondieren mit einem bestimmten Organ. Es gibt auch übergeordnete Punkte mit hormonähnlichen Wirkungen, medikamentenvergleichbare Punkte und psychisch wirksame Punkte.

Besonders bewährt hat sich die Ohrakupunktur bei folgenden Indikationen:

- akute und chronische Schmerzen und Erkrankungen des Bewegungsapparats: Zervikalgie, Lumbalgie, Ischialgie, Myalgien, Gelenkschmerzen etc.

- Kopfschmerzen, Migräne, Neuralgien

- funktionelle Störungen innerer Organe

- Allergien

- psychovegetative Befindlichkeitsstörungen

- Schwindel

- Schlafstörungen

- Suchttherapie

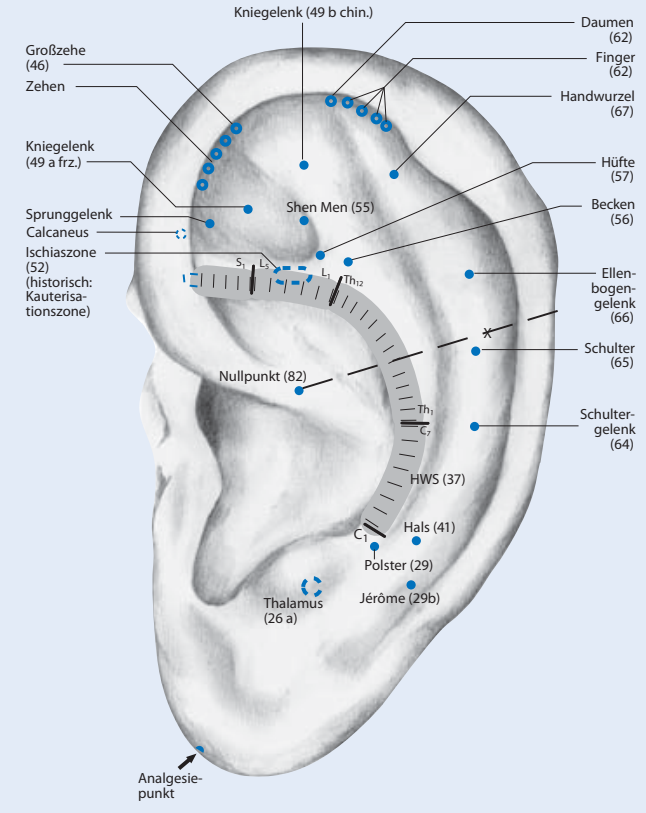

Abb. 3 Wichtige Ohrakupunkturpunkte des Bewegungsapparats. (c) [2]

Kontraindikationen sind gravierende Infektionserkrankungen, schwere neurologische Erkrankungen, Entzündungen am Ohr, Gerinnungsstörungen. Während der Schwangerschaft sind Punkte mit endokriner Wirkung zu vermeiden.

Cave: Hoch sensible Punkte sind nach Nogier zunächst nur zu beobachten und nicht zu nadeln.

\section{Erkrankungen des Bewegungsapparats}

Erkrankungen des Bewegungsapparats ( Abb. 3) sprechen sehr gut auf die Ohrakupunktur an. Doch ist darauf hinzuweisen, dass ein Herd- oder Störfeldgeschehen die Akupunkturwirkung behindern kann. Durch Herde oder Störfelder können körpereigenen Regulationssysteme instabil werden, geringfügige Reize werden inadäquat, also übertrieben, beantwortet. Für den Bewegungsapparat kommt es dabei zu einer unnötigen Überaktivität des neuromuskulären Systems in Form von pseudoradikulären Myospasmen und Schmerzen.

Ohrakupunktur und Körperakupunktur werden in diesen Fällen im günstigsten Fall nur eine vorübergehende Linderung der Beschwerden erreichen: Bereits lokale oder geringe allgemeine Reize können ein Wiederaufklingen der Beschwerden be- wirken, da die Ursache der Regulationsstörung bei der Therapie unberücksichtigt blieb. In diesen Fällen sollte eine Herdsanierung veranlasst oder zumindest neuraltherapeutisch eine Störfeldtherapie vorgenommen werden.

Beim chronisch rezidivierenden Geschehen haben sich 2 Sitzungen/Woche bewährt, bei gutem Ansprechen kann die Behandlung auf 1-wöchentlich reduziert werden. Es sind wiederholte Behandlungsserien von 10-15 Behandlungen notwendig, die sinnvollerweise in Kombination mit einer gezielten, z. B. eine Fehlhaltung korrigierenden Bewegungstherapie (Rolfing, Feldenkrais o.ä.) durchgeführt werden sollten. Erfahrungsgemäß ermöglicht die Ohrakupunktur überhaupt erst die Durchführung von Bewegungstherapien.

elda

\section{Verwendete Literatur}

[1] Ogal HP, Kolster BC, Hrsg. Ohrakupunktur für Praktiker. Grundlagen, Praxis, Indikationen. Stuttgart: Hippokrates; 2003

[2] Rubach A, Hrsg. Propädeutik der Ohrakupunktur. 3. Aufl. Stuttgart: Hippokrates; 2009

[3] Strittmatter B, Hrsg. Taschenatlas Ohrakupunktur nach Nogier/Bahr. Grundlagen, Lokalisationen, Indikationen. 4. Aufl. Stuttgart: Hippokrates; 2008 